\title{
Design and Implementation of Three Phase Multi-function Electric Parameters Measuring Meter Based on IDT_90E36
}

\author{
Nan $\mathrm{Xie}^{1, \mathrm{a}^{*}}$ and Bin $\mathrm{Xu}^{1, \mathrm{a}}$ \\ 1 Zhejiang University of Water Resources and Electric Power, Hangzhou, China \\ a905321899@qq.com \\ * The Corresponding Author
}

Keywords: IDT_90E36; STM32 MCU; Electric parameters; Electric measuring meter

\begin{abstract}
With the rapid development of smart grid, the accurate measurement of all kinds of linear and nonlinear loads' electrical parameters is a major problem facing us in the power grid system. The main chips of the electric measuring meter are STM32 serial microprocessor core and IDT_90E36 electric energy metering chip. The meter's measuring flow, hardware circuit design and the IDT_90E36 chip setting have been introduced in detail. Also, the use of BY2086 three-phase precise harmonic source as standard source as the evaluation index could realize the accurate measurement of power system load three-phase electrical parameters, and could load the harmonics generated by the nonlinear energy analysis special inspection. Finally, a prototype has been made and its electric parameters measurement accuracy is about 5/1000. The test results prove the feasibility of the meter design and the electric parameters measuring meter also improves the measurement accuracy and stability of electric parameters, and greatly simplifies the design of hardware circuit structure, reduces the cost of enterprise, and it has a market value of promotion and application.
\end{abstract}

\section{Introduction}

With the rapid development of power technology, various kinds of linear nonlinear loads have appeared in the power grid system. The measurement of various electrical parameters has become the main problem that we are facing. In such a broad market prospects, all kinds of electric energy meter also came into being [1]. At present, the existing watt hour meter has three phase electric energy meter based on ATT7021, CS5460 based electronic energy meter, and ADE7756 based intelligent watt hour meter. There are some defects in these meters. CS5460 is a single-phase electric energy metering chip, the dynamic range in order to ensure that the error is less than $0.1 \%$ in the range of $300: 1$; ATT7021 is a single-phase electric energy metering chip, ensure the same error in the dynamic range of 500:1 to ADE7756; the performance is slightly better, the dynamic range is increased to $0.1 \%$ and 1000:1 to ensure the error; the above, all the chips are not able to detect harmonics caused by the nonlinear load analysis[2-3].

The multi-function electric meter based on IDT_90E36 not only has the widest dynamic range in the industry (6000:1), but also combines the proprietary temperature compensation to get the lowest temperature coefficient, which can maintain its excellent performance in various complex industrial environments. Not only that, in addition to the normal function of the general meter, but also for the harmonic pollution caused by the increasingly serious nonlinear devices, the chip has special functions for harmonic detection and analysis.

\section{Characteristics of IDT_90E36 Chip}

Multiphase active power meter and 2 stage Multiphase Reactive power meter can be used for $0.5 \mathrm{~s}$ or $1 \mathrm{~s}$ level.

In the dynamic range of $6000: 1$, the accuracy of active power is up to $0.1 \%$, and the accuracy of reactive power is up to $0.2 \%$. 
The measurement of electrical parameters: the effective voltage value of sinusoidal AC signal, the effective current value of sinusoidal AC signal, the average value of active power, reactive power and apparent power, and the reference error of frequency, power factor and phase angle is less than $0.5 \%$.

Active power, reactive power and apparent energy have independent energy registers. They are read out by pulse output or read from energy registers, and are applied to different devices.

The A/B/C three-phase and midline current sampling circuits have their own independent $A D$ sampling and different gain. Current sampling is carried out on a real current transformer or a Rogowski coil, and the $\mathrm{A} / \mathrm{B} / \mathrm{C}$ three-phase is sampled on a voltage divider or transformer.

Programmable work mode: normal mode, idle mode, current detection mode, partial detection mode.

The active energy of the fundamental harmonic wave has a special energy and power register.

For the 2-32 harmonic components, the total harmonic distortion rate and the function of the discrete DFT transform can be detected, and the results are stored in the readable register of SPI. The voltage and current of all phases are processed in the same period of time [4].

\section{Design of Electric Parameter Meter}

The main chips of the meter are the STM32 serial microprocessor and the IDT_90E36 electric energy metering chip, and the meter test flow is shown in Fig. 1. First of all, it is sampled by a sampling module, then the data will be transmitted to the IDT_90E36 analysis, and stored in each register, MCU is responsible for the control of metering chip operation process and the data read from the register for processing, to a display part. The key input and reset circuit also control the metering chip through MCU to get the data to be read or to realize the reset operation [5-8].

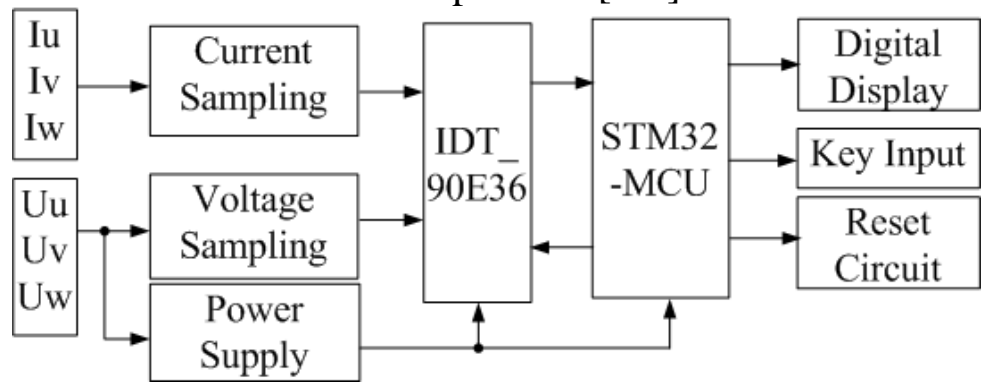

Figure 1. Multifunction meter measuring flow chart

\section{Hardware Circuit Design}

Sampling Circuit Design. Voltage sampling is based on resistor divider sampling, current sampling is sampled by current transformer, and $18 \mathrm{nF}$ capacitor filter is added at both ends of sampling and output, so that the sampling circuit has anti-aliasing effect.

The Connecting Part of MCU and IDT_90E36. All the sampled signals are processed by the DSP inside the metering chip IDT_90E36 to complete the accurate calculation of various parameters and be stored in the corresponding registers. MCU can realize data display, switch and so on by controlling the SDI of the metering chip and the reading and writing of the data at the SDO port.

\section{IDT_90E36 Setting}

Work Pattern Setting of IDT_90E36. Different modes of work correspond to different power consumption, and each mode of work corresponds to the function that is not used. Each mode of work is controlled by PM1/PM0. In the normal mode (PM1/PM0=11), the chip is in a normal working state, and all the registers of the chip can be accessed through the SPI. In the partial measurement mode (PM1/PM0 =10), only the current sampling related modules are in normal operation, and only registers related to current sampling can be accessed. In the current detection mode (PM1/PM0 $=01)$, all registers cannot be accessed, which can carry out SPI normal communication, but the current detection module is turned on, so the current detection and control registers need to register the threshold in normal mode is set, then enter the current detection mode and. In the idle mode (PM1/PM0=00), all the registers of the chip cannot be accessed, and all the functional modules are also closed [9-10]. 
When switching from idle mode to normal mode or partial measurement mode, registers need to be re loaded. Switching from idle mode to current mode does not need to reload registers.

In general, we set the work mode directly at the initial stage of the program in the software part, that is, the other $\mathrm{PM} 1 / \mathrm{PM} 0=11$, which is convenient to use.

Calibration of IDT_90E36. Three phase watt hour meter based on IDT_90E36 is designed. According to the chip instruction, we only need to calibrate active power, and other parameters need not be calibrated. Its accuracy is guaranteed by chip. The calibration flow of the chip is also described in order to calibrate the measured value in order to calibrate the measurement value first.

Firstly, the system software is reset. It is the register in the chip that can write or read the data effectively. Then the measurement / metering function is started, and the calibration command is written to the registers of ConfigStart, CalStart, HarmStart and AdjStart, so that the corresponding registers are reset to the default value. Secondly, the high frequency constant is set. The calculation of the electric energy in the metering chip is shown by the electric energy pulse CF. The relationship between the two is reflected by the constant of the meter. The set formula is: PL_constant=450,000,000,000/MC, the obtained value is converted to sixteen, and then stored in the high and low bit of its register respectively. Then the metering mode and the gain of the signal are set up. When setting the metering mode, the MMODE0 is set up according to the frequency of the power grid and the use range of the meter, and the MMODE1 is set according to the gain needed by the sampling and detection. After the completion of the setting is the school table. In accordance with the order of Figure 3.2, the values are written to the corresponding imbalance register, the gain register, the measurement value register, and the angle difference register. Finally, as the temperature characteristics of the components in the system will lead to temperature drift, some special registers are set up for temperature compensation.

Harmonic Analysis Setting. In order to accurately measure the harmonic, before the DFT transform, the first window of the wave is added to reduce the fence effect. Then set the pre amplification factor of the harmonic, because the harmonic signal is over hours, the error will increase. Next is the discrete DFT transform analysis, DFT began to write to the engine control command, because the analysis of a DFT, the engine will automatically shut down, so the detection of the DFT engine state value, we can judge whether the completion of the execution of DFT analysis. At last, the total harmonic distortion rate, the voltage value of the fundamental wave and the current value of the fundamental wave can be read from the corresponding register.

\section{Experiment and Result Analysis}

We can use the high precision standard source input, compare with the test value, and calculate the error to verify the feasibility of the design of the electric parameter meter. The standard source is BY2086 three-phase precision harmonic source, which can output the maximum range $380 \mathrm{~V}$ of the stable voltage, and the amplitude stability is $0.05 \%$, and the waveform distortion is $0.2 \%$. It can output a stable current of the maximum range rating of 50A, and the amplitude stability is $0.05 \%$, and the distortion of the waveform is $0.5 \%$. In addition, the harmonic source can output 2 21 harmonic, harmonic amplitude range: when $2 \sim 5=40 \%$; 6 15: when the time is less than or equal to 30\%; when the 16 21 is less than or equal to $10 \%$ times. Monitor dashboard instrument with a voltage meter, can show the actual output value or output value and the percentage of the selected range of the rated value rate, accuracy grade 0.5; current meter, can show the actual output value or output value and the percentage of the selected range of the rated value rate, accuracy class 0.5 . The electrical parameters measured on the basis of the standard source are shown in Table 1.

From Table 1, it can be seen that the precision of the design for the parameters of voltage, current and phase angle is about $5 / 1000$. The experiments show that the design of the meter is feasible and has good measurement accuracy and measuring stability. 
Table 1 Test data for the electric parameters meter

\begin{tabular}{|l|l|l|l|l|l|l|l|l|l|}
\hline $\begin{array}{l}\text { Measur } \\
\text { ement } \\
\text { Phase }\end{array}$ & $\begin{array}{l}\text { Voltage } \\
\text { input } \\
\text { value } \\
(\mathrm{V})\end{array}$ & $\begin{array}{l}\text { Voltage } \\
\text { measur } \\
\text { ement } \\
\text { value } \\
(\mathrm{V})\end{array}$ & $\begin{array}{l}\text { Voltag } \\
\text { e error } \\
(\%)\end{array}$ & $\begin{array}{l}\text { Current } \\
\text { input } \\
\text { value } \\
(\mathrm{A})\end{array}$ & $\begin{array}{l}\text { Current } \\
\text { Measu } \\
\text { rement } \\
\text { value } \\
(\mathrm{A})\end{array}$ & $\begin{array}{l}\text { Curre } \\
\text { nt } \\
\text { error } \\
(\%)\end{array}$ & $\begin{array}{l}\text { Phase } \\
\text { angle } \\
\text { setting } \\
\text { value }\left(^{\circ}\right)\end{array}$ & $\begin{array}{l}\text { Phase angle } \\
\text { measuremen } \\
\text { t value }\left(^{\circ}\right)\end{array}$ & $\begin{array}{l}\text { Phase } \\
\text { angle } \\
\text { error } \\
(\%)\end{array}$ \\
\hline & 379.8 & 380.1 & 0.08 & 4.998 & 5.00 & 0.04 & 30.0 & 30.1 & 0.33 \\
\hline & 369.9 & 369.6 & -0.08 & 4.003 & 4.01 & 0.17 & 40.0 & 40.0 & 0.00 \\
\hline & 359.4 & 359.2 & -0.06 & 2.997 & 2.98 & -0.56 & 50.0 & 49.9 & -0.33 \\
\hline & 349.2 & 348.7 & -0.14 & 1.996 & 1.97 & -1.30 & 60.0 & 59.8 & -0.33 \\
\hline & 378.9 & 379.6 & 0.18 & 4.998 & 5.01 & 0.24 & 330 & -29.9 & -0.33 \\
\hline & 368.5 & 368.2 & -0.08 & 3.996 & 3.98 & -0.40 & 320 & -40.1 & 0.25 \\
\hline & 358.3 & 357.9 & -0.11 & 3.001 & 2.99 & -0.37 & 310 & -49.6 & -0.80 \\
\hline & 348.2 & 347.7 & -0.14 & 1.997 & 1.98 & -0.85 & 300 & -59.5 & -0.83 \\
\hline
\end{tabular}

\section{Conclusions}

The application of STM32 serial microcontroller and IDT_90E36 chip energy metering completes the design of measurement system for three-phase power, performance characteristics of the electric energy metering chip key circuit structure, measurement chip calibration is described in detail, and the use of BY2086 three-phase precise harmonic source as standard source as the evaluation index, the three-phase the electric voltage, current, phase, power, harmonic active power, power factor, power capacity and other parameters with accurate measurement. This design has been carried out sample trial, the test results show that the intelligent electrical parameters measuring meter measurement accuracy and stable performance, draft design could simplify the hardware circuit and software structure, save the cost, shorten the development cycle, and have a special analysis function of the harmonics generated by nonlinear loads. If the meter design has improved and join remote communication module, it would enter the production stage and have very broad application prospects.

\section{Acknowledgements}

This work was supported by the 2017 New Talents Project of Zhejiang Province, China (Project CODE: 2017R434001).

\section{References}

[1] Electrical measurement equipment (AC) of the International Electrotechnical Commission (AC). Special requirements. Twenty-first: static watt hour meter (Level 1 and 2): IEC 62053-21[S]

[2] Peng L, Shizhao N, Zheng W, Ziwei J, Jianwu Y, Zhongxiang Q, Wangmo P. Predicting durations of online collective actions based on Peaks' heights [J]. Communications in Nonlinear Science and Numerical Simulation. 2018, 55: 338-354.

[3] Gan Jianping, Zhu Qing. Design of Watt-hour Meter Based on Energy IC CS5463A [J]. China Instrumentation, 2007(04): 39-42.

[4] Chen Yake, Cao Naochang, Wang Jiaxiang, etc. Design of the Energy Meter Based on ADE7753 and MSP430 [J]. Process Automation Instrumentation, 2011(04): 66-70.

[5] Design of IDT90E36 three-phase power meter, http://tech.hqew.com/fangan_756807, 2016.8.10.

[6] Zhao Hongshan, Mi Zengqiang, Wang Haiping. A intelligent watt hour meter based on IÇ15 ADE7756 [J]. Electrical Measurement \& Instrumentation , 2001(09): 48-50. 
[7] Xu Jingsheng, Nie Jianbo, Dong Zhanguo, etc. Low-power Long-distance Wireless Transmission Smart Meter based on HT6015 [J]. Instrumentation· Analysis· Monitoring, 2017(04): 8-13.

[8] Zhang Xin. Design and Research of Smart Meter Based on MSP430 [J]. Industrial Control Computer, 2017(09): 117-119.

[9] Research on the key technology of the dual MCUs on the energy meter for the IR46 standard [J], Application of Electronic Technique, 2017(10): 7-12.

[10] Xia Wen.Verilog digital system design tutorial [M]. Beijing: Beihang University Press, 2003.

[11] Huang Yizhuang. Substation integrated automation technology [M]. China Electric Power Press, 2000. 